\title{
Design optimization of industrial gas turbines using simulated annealing algorithms
}

\author{
Mohammadreza $\operatorname{Tahan}^{1}$, A.L.Tamiru ${ }^{1, ~ *}$, and Masdi Muhammad ${ }^{1}$, \\ ${ }^{1}$ Department of Mechanical Engineering, Universiti Teknologi PETRONAS, 32610 Bandar Seri \\ Iskandar, Perak Darul Ridzuan, Malaysia.
}

\begin{abstract}
Currently, gas turbine is one of the most widely-used power generating technologies. The race to achieve higher efficiency from gas turbines is gathering momentum with most of the major manufacturers. Cogeneration with advanced engines has the prospect of attaining thermal efficiencies around $60 \%$ in the future. In this condition, further development of gas turbine design optimization in order to obtain higher thermal efficiency seems to be beneficial. In the current work, the design of a single shaft gas turbine in a cogeneration plant is optimized based on the model established using thermodynamic theory. The overall thermal efficiency of the engine is tried to be optimized by adjusting the compressor efficiency, turbine efficiency, compression pressure ratio, and turbine inlet temperatures. A feasible solution should satisfy two physical constraints, namely a desired gas turbine power and a suitable limit of engine exhaust temperature. An evolutionary model using Simulated Annealing algorithm is developed to find the sets of optimal solutions in the space defined by user experience and literature. A number of case studies have been performed and an optimal solution and their corresponding performance are discussed
\end{abstract}

\section{Introduction}

Industrial gas turbine (IGT) is related to the majority of energy consumption in the world. IGTs play a central role in power generation, because of their relatively low installed capital cost, their high flexibility, and their low emissions with respect to other energy conversion systems [1]. Due to the increasing global demand, they are still expected to continue playing a major role in energy production market.

Open-cycle gas turbine engines exhaust a high-grade heat to the atmosphere. The cogeneration (cogen) through combined heat and power (CHP) is the simultaneous production of electricity with the recovery and utilization of the exhaust heat from the gas turbine engine. Gas turbine as the main equipment of gogeneration power generation are typically manufactured in two basic configurations, single-shaft and multi-shaft. Single shaft gas turbine units feature direct mechanical coupling between power turbine stages and compressor drive turbine stages so that all rotating elements operate at the same speed. This model has been applied to power generation and other suitable constant speed applications

\footnotetext{
*Corresponding author: tamiru.lemma@utp.edu.my
} 
where rotating mass inertia is important for operating stability. Consequently, most large industrial turbines are of single shaft design.

Cogeneration is a highly efficient form of energy conversion and it can achieve primary energy savings of approximately $40 \%$ by compared to the separate purchase of electricity from the national electricity grid and a gas boiler for onsite heating. However, cogeneration plants can be even more efficient if thermal optimization being employed. Thermal optimization has two branches: system optimization and component optimization. The first refers to overall thermal systems and the second to the individual components such as gas turbine that make up the overall systems.

The objective of the presented paper is to assess a thermal design optimization of the single shaft gas turbine engine installed in a cogeneration plant. For this purpose, first, a thermodynamic model of the gas turbine is developed. Then using one of the most common metahuristic algorithms know as Simulated Annealing (SA), the optimum design parameters to achieve the desired output power and exhaust temperature with the highest overall thermal efficiency are obtained.

\section{Gas turbine design points modeling}

The case study is a Solar Taurus 60S gas turbine based cogeneration plant. The schematic diagram of this plant is shown in Figure 1. The Taurus $60 \mathrm{~S}$ has an ISO rating power production capacity of 5.3 MW (5 MW generator terminal power). The compressor and turbine design point data are calculated using energy and mass conversions and thermodynamic property ratio relationships. To proceed along the path followed by the working fluid, the following modeling assumptions are made.

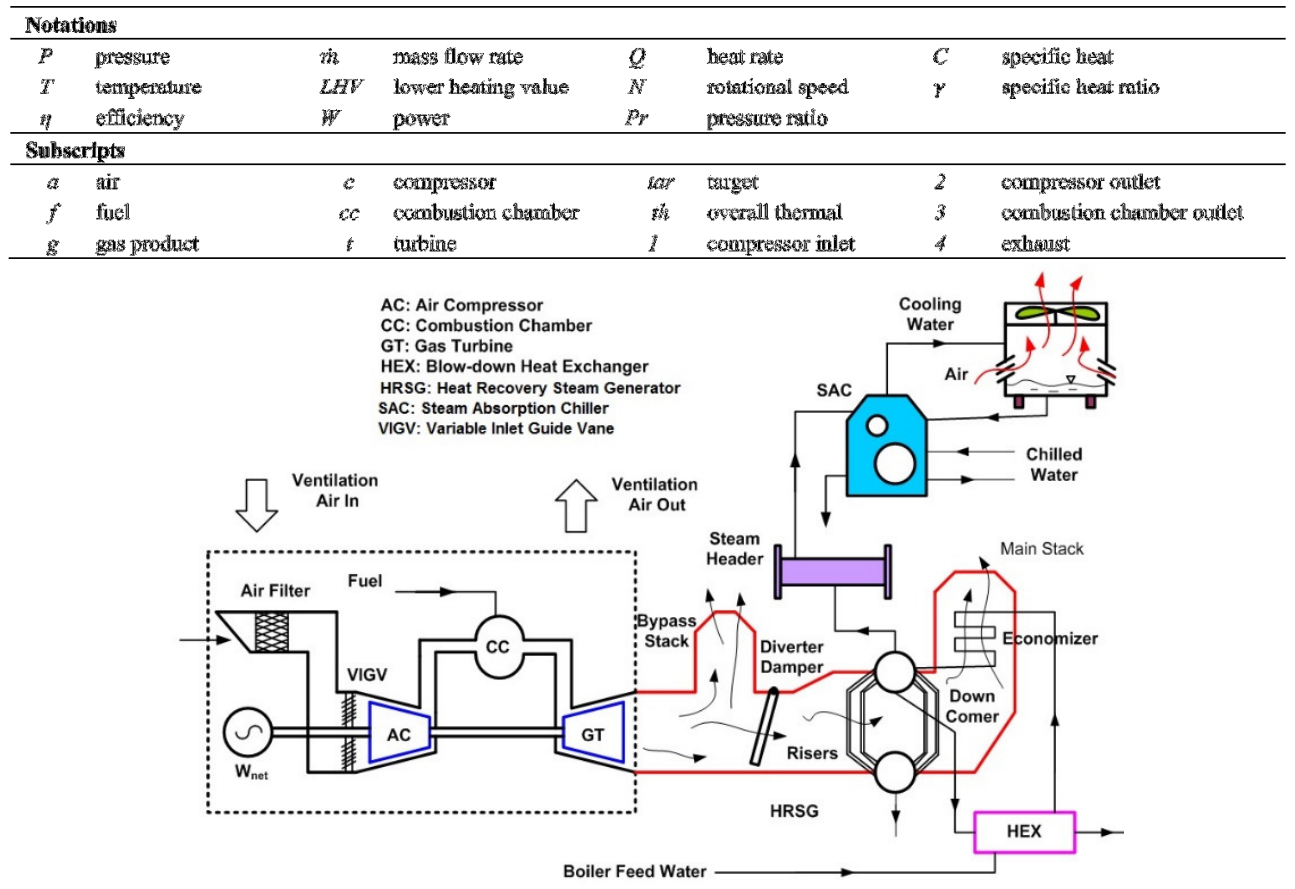

Fig. 1. Schematic diagram of the studied cogeneration plant.

All the calculations are done at the mean site ambient condition, i.e. $298.15^{\circ} \mathrm{C}$ and 1 atm pressure.

- Ideal-gas principles are applied for the air, fuel, and the combustion products. 
- The inlet air is assumed to be dry air and the considered fuel is methane having the lower heating value of $50 \mathrm{MJ} / \mathrm{kg}$.

- The percentage of combustion chamber pressure loss and exit pressure loss, including preheater and HRSG system, is assumed to be $5 \%$ and $8 \%$, respectively.

First, the compressor exit temperature is calculated using Equation (1).

$$
T_{2}=T_{1}\left(1+\frac{1}{\eta_{c}}\left[\operatorname{Pr}_{c}^{\frac{\gamma(T)-1}{\gamma(T)}}-1\right]\right)
$$

where $T, p$, and $\eta_{p}$ are the temperature, pressure, and compressor isonteropic efficiency, respectively. $\gamma$ is the specific heat ratio and is dependent to air temperature. Here, this temperature is considered the mean of $T_{1}$ and $T_{2}$. Once the compressor exit temperature is known, the power input to the compressor can be calculated by Equation (2).

$$
W_{c}=\dot{m}_{a} C_{p a}\left(T_{2}-T_{1}\right)=\dot{m}_{a} \int_{T_{1}}^{T_{2}} C_{p}(T) d T
$$

Combustion charts can be employed to calculate the fuel flow using combustor inlet temperature and estimated turbine inlet temperature [2]. The heat input to the combustion chamber is therefore given by:

$$
Q_{c c}=\dot{m}_{f} \times L H V
$$

Then, the turbine exit temperature can be achieved using Equation (4).

$$
T_{4}=T_{3}\left(1-\eta_{t}\left[1-\left(\frac{1}{P r_{t}}\right)^{\frac{\gamma(T)-1}{\gamma(T)}}\right]\right.
$$

where $\eta_{t}$ is the turbine isonteropic efficiency and $\gamma$ is the specific heat ratio. which is dependent to air temperature. Here, this temperature is considered the mean of $T_{3}$ and $T_{4}$.

And finally the power produced by the turbine, the net power output and the engine thermal efficiencies are computed by:

$$
\begin{aligned}
& W_{t}=\dot{m} C_{p g}\left(T_{3}-T_{4}\right)=\dot{m}_{g} \int_{T_{3}}^{T_{4}} C_{p}(T) d T \\
& W_{n e t}=W_{t}-W_{c} \\
& \eta_{t h}=\frac{W_{n e t}}{Q_{c c}}
\end{aligned}
$$

\section{Evolutionary algorithms}

Finding an optimal solution for certain optimization problems can be an incredibly difficult task, often practically impossible. This is because when a problem gets sufficiently large, we need to search through an enormous number of possible solutions to find the optimal one. Even with modern computing power, there are still often too many possible solutions to consider. In this case, because we can't realistically expect to find the optimal one within a sensible length of time, we have to settle for something that's close enough. Evolutionary algorithms (EIs), which are generic population-based metaheuristic optimization algorithms, often perform well approximating solutions to all types of problems. 
Simulated annealing (SA) is one of evolutionary optimization algorithms which its main strength is avoiding of getting caught at local solutions that are better than any others nearby. SA injects just the right amount of randomness into things to escape local maxima early in the process without getting off course late in the game when a solution is nearby. This makes it pretty good at tracking down a decent answer, no matter its starting point.

Simulated annealing (SA) is a metaheuristic approach for approximating the global optimization in a large search space. In this method, the Metropolis Algorithm is employed to simulate annealing in metallurgy, a technique involving controlled heating and cooling of a material to increase the size of its crystals and reduce its defects. By repeatedly observing this Metropolis rule of acceptance, a sequence of configurations is generated, which constitutes a Markov chain. A finite time implementation of the SA algorithm can be realized by generating homogeneous Markov chains of finite length, $M$, for a finite sequence of descending values of temperature, $\mathrm{T}$.

Each iteration of SA algorithm attempts to replace the current solution $\left(X_{i}\right)$ with a random solution that is chosen from its neighborhood $\left(X_{i+1}\right)$. The new solution is accepted if it improves the objective function $(E)$ or with a probability $(e)$ that depends both on the difference between the objective function values $(\Delta E)$ and also on annealing temperature $(T)$, which is gradually decreasing based on annealing schedule [3]. Figure 2 shows the principles of this optimization algorithm.

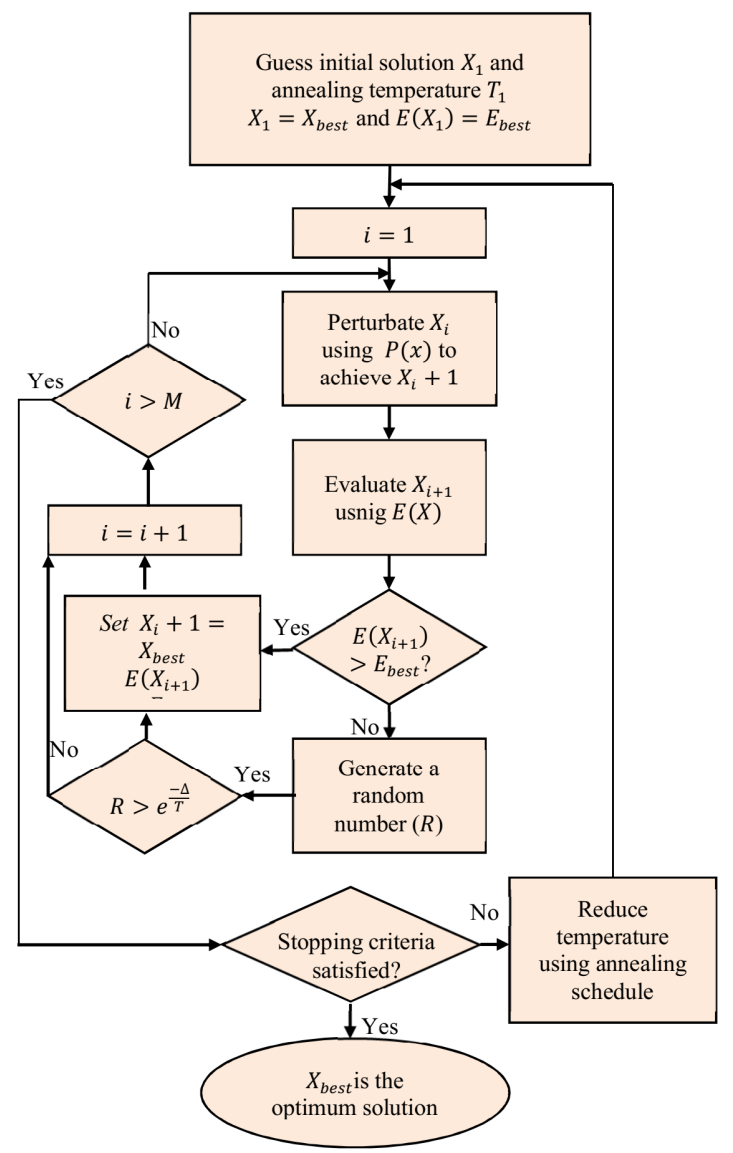

Fig. 2. Schematic diagram of the experiment setup. 
The detail of this technique is described in below.

1. Set $i=1$.

2. Guess the initial design variable vector $X_{i}$, eveluate it using objective function $E$, set $X_{i}$ as $X_{\text {best }}$ and $E\left(X_{i}\right)$ as $E_{\text {best }}$.

3. Select initial temperature for simulated annealing algorithm, and also outline the annealing schedule.

4. Perturb $X_{i}$ using $P(x)$ to obtain a neighboring design vector $\left(X_{i+1}\right)$.

5. Evaluate $E\left(X_{i+1}\right)$ using obojective function.

6. If $E\left(X_{i+1}\right)>E_{\text {best }}, X_{i+1}$ is the new solution and $E\left(X_{i+1}\right)$ need to be set as $E_{\text {best }}$.

7. If $E\left(X_{i+1}\right)<E_{\text {best }}$, then accept $X_{i+1}$ as the new solution with a probability $e\left(X_{i+1}\right)=e^{\frac{-\Delta \mathrm{E}}{T}}$ where $\Delta E=E\left(X_{i+1}\right)-E\left(X_{i}\right)$.

8. Set $i=i+1$ and repeat Step 4 to 8 if $M<i$.

9. Reduce $T$ using the annealing schedule.

10. Repeat steps 3 to 10 until the stopping criterion is fulfilled.

\section{The layout of the optimization model}

The modeling of every optimization problem involves selection of the design parameters, the objective functions and the constraints of the problem.

The goal of the optimization algorithm is to maximize engine thermal efficiency. Therefore, the problem objective function can be adjusted as Equation (8).

$$
f_{1}=\eta_{t h}(x)
$$

Along with the objective function, the constraints of the problem need to be defined clearly. In our case study model, the following two considerations have been done:

- The exhaust gas temperature which is conducted through a heat recovery steam generator (HRSG) system to produce medium to high temperature process steam should be in the range of $723.15-873.15 \mathrm{~K}$. This constrained can be expressed as follows:

$$
723.15 \mathrm{~K}<T_{4}<873.15 \mathrm{~K}
$$

- The desired produced electrical power needs to be $4.7 \mathrm{MW}$. The gearbox coupling efficiency and the generator power conversion efficiency are considered $98.2 \%$ and $96.4 \%$, respectively. Therefore, the engine thermodynamic power output should be around $5 \mathrm{MW}$. This constraint is applied as follows:

$$
W=5 \pm \delta \mathrm{MW}
$$

where $\delta$ is the maximum allowable inaccuracy, and here, is considered to be $10^{-4}$. The defined objective function and constraints greatly depend on the efficiency of the component efficiencies, maximum cycle temperature, and compression ratio. Therefore, here, four variables are considered as optimization design parameters including axial compressor pressure ratio, the turbine inlet temperature, the compressor isentropic efficiency, and turbine isentropic efficiency. The first two design parameters can change within the optimization interval according to previously established limits. However, the two former ones may be set constant in some optimization trials or similar to the previous ones change within the optimization interval according. Table 1 shows the most appropriate range for each parameter. Most of these limits are determined according to the authors experience or base on some reference documents including $[4,5]$. 
Table 1. Limit values of the design variables.

\begin{tabular}{|c|c|c|}
\hline Design variable & Minimum & Maximum \\
\hline $\boldsymbol{P}_{\boldsymbol{c}}$ & 6 & 15 \\
\hline $\boldsymbol{T}_{\mathbf{4}}$ & 900 & 1500 \\
\hline $\boldsymbol{\eta}_{\boldsymbol{c}}$ & 0.78 & 0.86 \\
\hline $\boldsymbol{\eta}_{\boldsymbol{t}}$ & 0.80 & 0.91 \\
\hline
\end{tabular}

As stated earlier, in some cases the value of $\eta_{\mathrm{c}}$ and $\eta_{\mathrm{t}}$ are considered constant and equals to the maximum of limits, i.e 0.86 and 0.91 for compressor and turbine efficiencies respectively.

In fact, the focus of this study is in determining the optimal design parameters that allow the gas turbine to operate at a specific power and exhaust temperature with the highest efficiency. This optimization problem can be characterized in the form of :

Maximize: $f_{1}=\eta_{t h}(x)$

Subject to:

$$
\begin{aligned}
& g_{1}=\left[\operatorname{abs}\left(W_{\text {tar }}(x)-W_{\text {net }}(x)\right)-\delta\right] \leq 0 \\
& g_{2}=\left[T_{4}-723.15\right] \geq 0 \\
& g_{3}=\left[T_{4}-873.15\right] \leq 0
\end{aligned}
$$

Where: $\boldsymbol{X}=\left[\operatorname{Pr}_{c}, T_{4}, \eta_{c}, \eta_{t}\right]$

$$
\begin{gathered}
6 \leq X(1) \leq 15 \\
900 \leq X(2) \leq 1500 \\
0.78 \leq X(3) \leq 0.86 \\
0.8 \leq X(4) \leq 0.91
\end{gathered}
$$

\section{Results and discussions}

Using the optimization model developed, two separate case studies have been performed.

Case study 1) At the first case study, compressor and turbine efficiencies are assumed to be constant. These variables are set to their maximum values which are $86 \%$ and $91 \%$, respectively. In this case, the optimization algorithm was performed to find the sets of other design variables, i.e. compressor pressure ratio and turbine inlet temperature in a way that maximize the overall engine thermal efficiency. The optimization algorithm is run for 100 times and it has found that 44 distinguished sets of parameters can fully satisfy the constraints. Table 2 shows some sample results of this case study.

Table 2. Sample results of the first case study.

\begin{tabular}{|l|c|c|c|}
\hline Variable & $\begin{array}{c}\text { Sample } \\
\text { point 1 }\end{array}$ & $\begin{array}{c}\text { Sample } \\
\text { point 2 }\end{array}$ & $\begin{array}{c}\text { Sample } \\
\text { point 3 }\end{array}$ \\
\hline $\boldsymbol{T}_{\mathbf{4}}(\mathbf{K})$ & 723.75 & 783.53 & 877.20 \\
\hline $\boldsymbol{\eta}_{\boldsymbol{t h}}(\boldsymbol{\%})$ & 0.3392 & 0.3074 & 0.2674 \\
\hline $\boldsymbol{P r}_{\boldsymbol{c}}$ & 12.78 & 8.81 & 6.005 \\
\hline $\boldsymbol{T}_{\mathbf{3}}(\mathbf{K})$ & 1227.48 & 1224.1 & 1239.2 \\
\hline $\boldsymbol{\eta}_{\boldsymbol{c}}(\boldsymbol{\%})$ & 86 & 86 & 86 \\
\hline $\boldsymbol{\eta}_{\boldsymbol{t}}(\boldsymbol{\%})$ & 91 & 91 & 91 \\
\hline
\end{tabular}


Where in this table, sample point 1 corresponds to best efficiency point, sample point 2 corresponds to the point with most desirable exhaust temperature, i.e. $783.15 \mathrm{~K}$, and sample point 3 corresponds to the point with minimum efficiency.

As can be seen, the best efficiency is achievable when the exhaust temperature is close to lower limit of the constraint stated in Equation (9). In the other obtained optimum design sets, even though the turbine outlet temperature is within the acceptable limit, bu it may be higher up to the $873.15^{\circ} \mathrm{K}$. The 44 obtined optimum sets are sorted base on maximum to minimum overall engine thermall efficiency and are shown in Figures 3(a)-3(b). The corresponding values of turbine thermal efficiency and maximum cycle temperature are shown in Figures 3(c)-3(d).

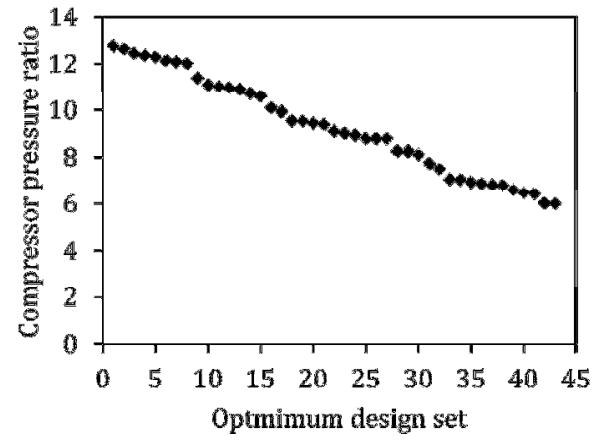

(a)

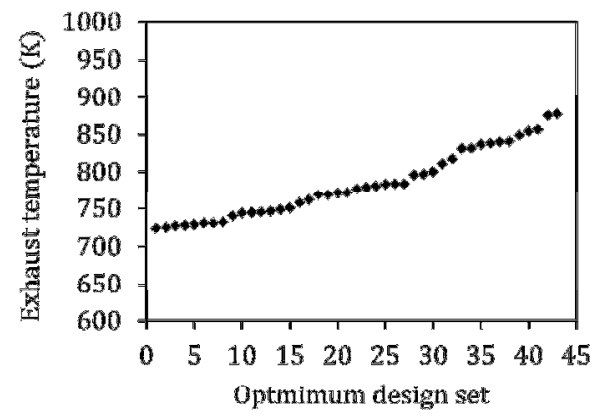

(c)

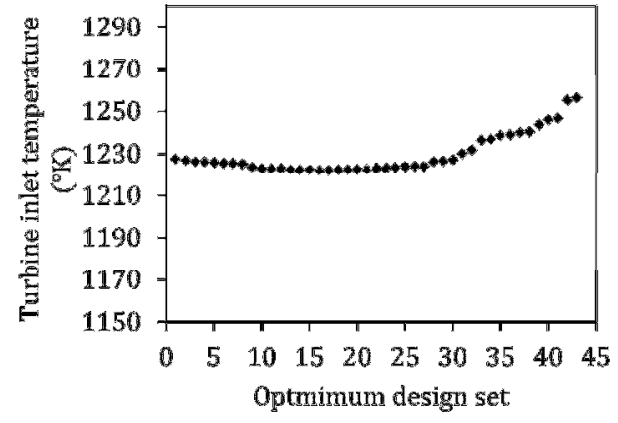

(b)

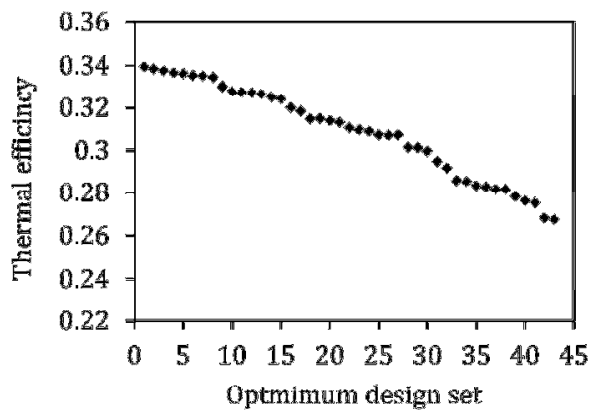

(d)

Fig. 3. Case study 1: the 44 obtained optimum design sets and their corresponding performance. (a) Compressor pressure ratio as the first design variable. (b) Turbine inlet temperature as the second design variable. (c) Gas turbine exhaust temperature corresponding to obtained design sets. (d) Gas turbine thermal efficiency corresponding to obtained design sets.

As can be seen in this graphs, to generate a constant power at the design point, the compressor pressure ratio needs to be decreased in order to achieve higher exhaust temperature. At the same time, the turbine inlet temperature should be increased which means more fuel flow will be consumed. This will consequently lead to lower overall thermal efficiency. Based on the graphs, it can be said that increasing the exhaust temperature up to $800^{\circ} \mathrm{K}$ seems to be to some extent economical, but over that will causes very low thermal efficiencies and need to be considered along with other decision parameter

Case study 2) At the second case study, design variables are increased to four parameters including compressor pressure ratio, turbine inlet temperature, compressor efficiency, and turbine efficiency. The optimization algorithm is again run for 100 times and this time, 63 distinguished sets of parameters are found that can fully satisfy the constraints. Table 3 shows some sample results of the second case study. Where similar to Table 2 , sample point 1 corresponds to best efficiency point, sample point 2 corresponds to the point with most 
desirable exhaust temperature, i.e. $783.15 \mathrm{~K}$, and sample point 3 corresponds to the point with minimum efficiency.

Table 3. Sample results of the second case study.

\begin{tabular}{|l|c|c|c|}
\hline Variable & $\begin{array}{c}\text { Sample } \\
\text { point 1 }\end{array}$ & $\begin{array}{c}\text { Sample } \\
\text { point 2 }\end{array}$ & $\begin{array}{c}\text { Sample } \\
\text { point 3 }\end{array}$ \\
\hline $\boldsymbol{T}_{\mathbf{4}}\left({ }^{\circ} \mathbf{K}\right)$ & 733.40 & 782.19 & 853.60 \\
\hline $\boldsymbol{\eta}_{\boldsymbol{t h}}(\boldsymbol{\%})$ & 0.3345 & 0.3092 & 0.2782 \\
\hline $\boldsymbol{P r}_{\boldsymbol{c}}$ & 14.22 & 12.583 & 13.0201 \\
\hline $\boldsymbol{T}_{\mathbf{3}}\left({ }^{\circ} \mathbf{K}\right)$ & 1262.35 & 1285.33 & 1351.63 \\
\hline $\boldsymbol{\eta}_{\boldsymbol{c}}(\boldsymbol{\%})$ & 83.58 & 83.49 & 84.14 \\
\hline $\boldsymbol{\eta}_{\boldsymbol{t}}(\mathbf{\%})$ & 90.81 & 88.49 & 83.29 \\
\hline
\end{tabular}

All the 63 obtained optimum sets are sorted base on maximum to minimum overall engine thermal efficiency and are shown in Figures 4(a)-4(d). The corresponding values of turbine thermal efficiency and maximum cycle temperature are shown in Figures 4(e)-4(f).

Based on the obtained results, the following conclusions can be inferred.

- In the second case study, which compressor and turbine efficiencies can change, the required compression ratio have increased to around 14 compared to the first study where the compression ratio is less than 12.2.

- Higher exhaust temperature can be achieved at higher turbine inlet temperatures. This will consequently increase the fuel flow rate and decrease the overall thermal efficiency.

- In most obtained design sets, the required turbine efficiency is more than the required compressor efficiency. In addition, to increase the thermal efficiency, higher turbine efficiency is required. However, there is not a specific rule or trend for compressor efficiency.

The outcomes of case study 1 and 2 prove the effectiveness of the proposed developed evolutionary algorithm for finding the optimal design variables in an engine thermodynamic optimization problem. However, the following items may assist to achieve more accurate results:

- Specifying a more limited acceptable range of exhaust temperature will reduce the number of optimal design sets.

- In addition to thermodynamic analysis, economic and environmental studies need to be performed. This will ensure that the designed engine is optimum from various critical aspects. 


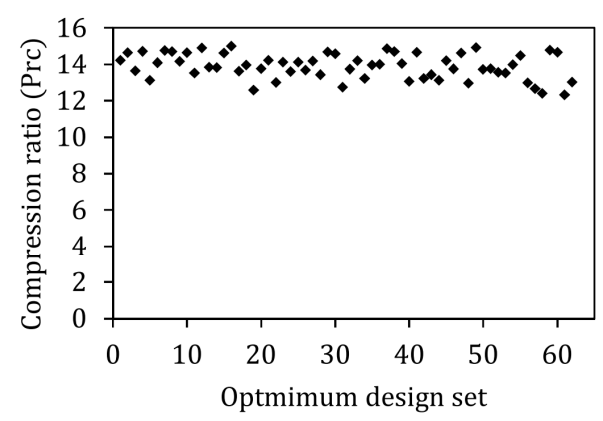

(a)

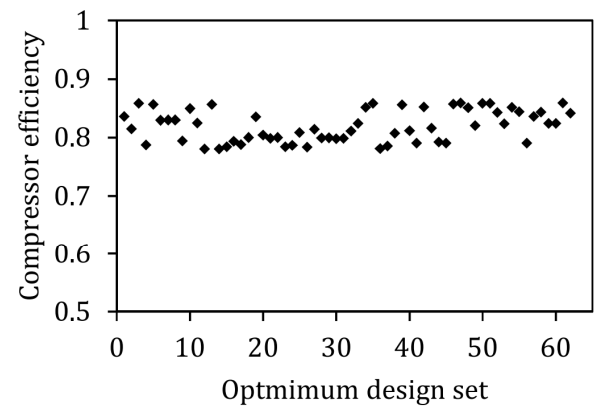

(c)

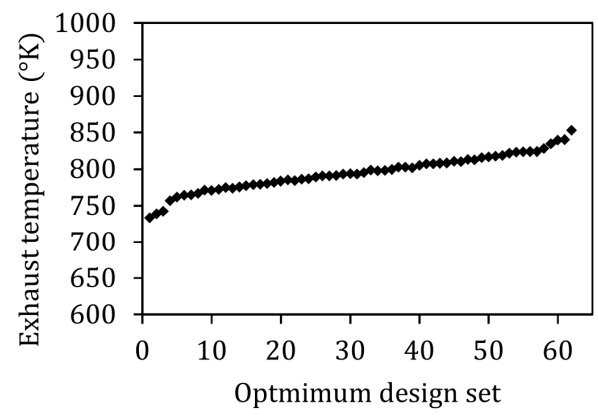

(e)

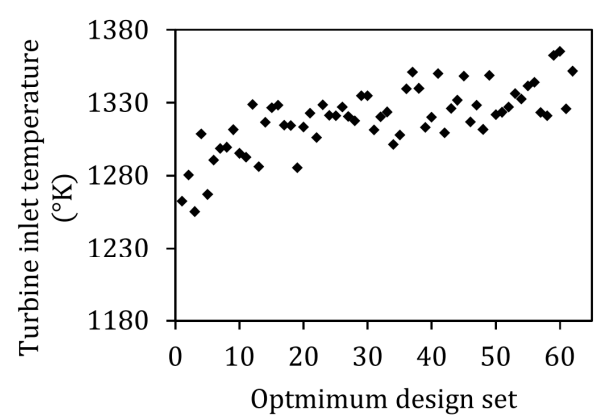

(b)

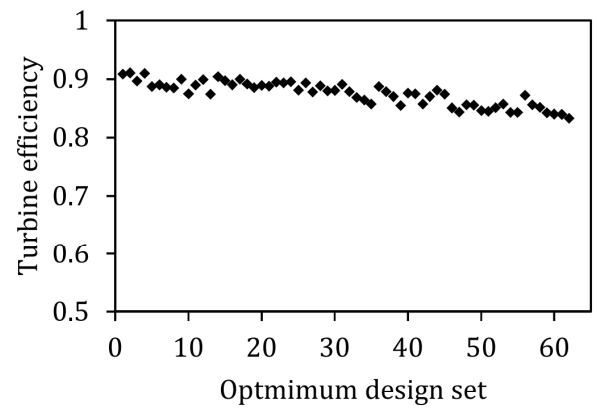

(d)

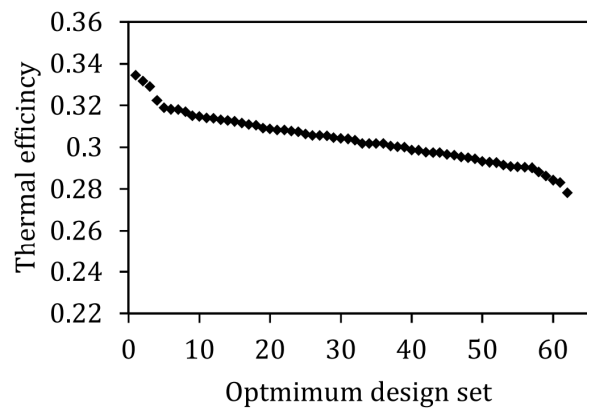

(f)

Fig. 4. Case study 2: the 63 obtained optimum design sets and their corresponding performance. (a) Compressor pressure ratio as the first design variable. (b) Turbine inlet temperature as the second design variable. (c) Compressor efficiency as the third design variable. (d) Turbine efficiency as the fourth design variable. (e) Gas turbine exhaust temperature corresponding to obtained design sets. (f) Gas turbine thermal efficiency corresponding to obtained design.

\section{Conclusion}

In this paper, a thermodynamic-based model for design point optimization of a gas turbine in a cogeneration plant is developed. The engine thermal efficiency as the objective function along with two main constraints including a constant desired power and an acceptable range of turbine exhaust temperature charactrize the optimization problem. The optimum design sets can be obtained by accounting for the interplay between the four design variables. Toward this end, a simulated annealing based optimization model is developed and its capability is examined through two case studies. 
- The first case study shows when the compressor and turbine efficiencies are considered constants to their maximum values, i.e. 0.86 and 0.91 respectively, the maximum obtainable thermal efficiency is $33.92 \%$ that can be achieved when the compression ratio is 12.78 , turbine inlet temperature is $1227.48^{\circ} \mathrm{K}$ and exhasut temperature is $723.75^{\circ} \mathrm{K}$.

- In the next case study, the maximum obtainable thermal efficiency is $33.45 \%$ by the design variable of $83.58 \%$ compressor efficiency, $90.81 \%$ turbine efficiency, 12.78 compression ratio, $1262.35^{\circ} \mathrm{K}$ turbine inlet temperature and $733.40{ }^{\circ} \mathrm{K}$ exhasut temperature.

The results obtained through two case studies proved the effectiveness of the proposed method for thermodynamic design optimization of gas turbine engines. This study can be extended further to optimize economic and environmental issues along with the thermodynamic performance.

The authors are grateful for the funding and facilities support by Universiti Teknologi PETRONAS.

\section{References}

1. M. Tahan Bouriaabadi, M. A. bin Abd Majid, A. Majid, M. Amin, and M. Muhammad, Appl. Mech. Mater., 786, 238 (2015).

2. P. P. Walsh and P. Fletcher, Gas turbine performance, (John Wiley \& Sons, 2004).

3. C. Wang, D. Mu, F. Zhao, and J. W. Sutherland, Comput. Ind. Eng., 83, 111 (2015).

4. I. J. Nickerson, A parametric study of gas turbine cycles for electrical power generation, (Carleton University, 1995).

5. G. L. Converse and R. Giffin, Extended parametric representation of compressor fans and turbines. Volume 1: CMGEN user's manual, (1984). 